\title{
FLOWTRAN-TF Code Modifications made for APT Blanket Safety Analyses
}

by

L. L. Hamm

Westinghouse Savannah River Company

Savannah River Site

Aiken, South Carolina 29808

S. Y. Lee

M. A. Shadday

F. G. Smith, III

A document prepared for SENDING TO LANL at , , from - .

DOE Contract No. DE-AC09-96SR18500

This paper was prepared in connection with work done under the above contract number with the $U$. S.

Department of Energy. By acceptance of this paper, the publisher and/or recipient acknowledges the U. S. Government's right to retain a nonexclusive, royalty-free license in and to any copyright covering this paper, along with the right to reproduce and to authorize others to reproduce all or part of the copyrighted paper. 


\section{FLOWTRAN-TF CODE MODIFICATIONS MADE FOR APT BLANKET SAFETY ANALYSES}

L. Larry Hamm

Si Young Lee

M. Andy Shadday

Frank G. Smith, III

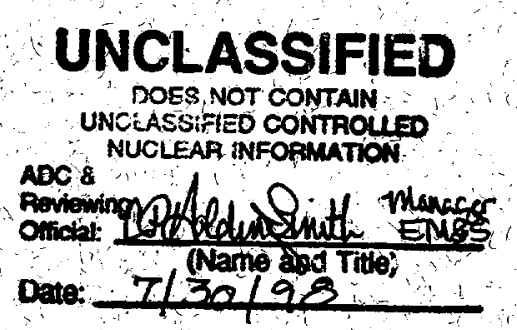

Westinghouse Savannah River Company

Savannah River Site

Aiken, SC 29808

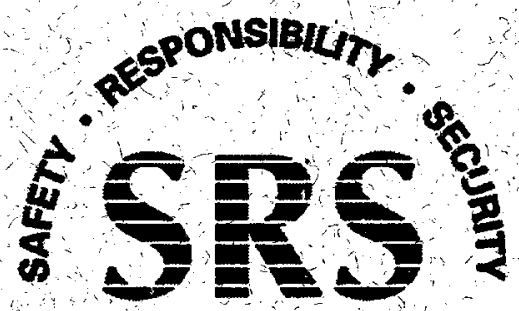




\section{DISCLAIMER}

This report was prepared as an account of work sponsored by an agency of the United States Government. Neither the United States Government nor any agency thereof, nor any of their employees, makes any warranty, express or implied, or assumes any legal liability or responsibility for the accuracy, completeness, or usefulness of any information, apparatus, product, or process disclosed, or represents that its use would not infringe privately owned rights. Reference herein to any specific commercial product, process, or service by trade name, trademark, manufacturer, or otherwise does not necessarily constitute or imply its endorsement, recommendation, or favoring by the United States Government or any agency thereof. The views and opinions of authors expressed herein do not necessarily state or reflect those of the United States Government or any agency thereof.

This report has been reproduced directly from the best available copy.

Available to DOE and DOE contractors from the Office of Scientific and Technical Information, P.O. Box 62, Oak Ridge, TN 37831; prices available from (615) 576-8401.

Available to the public from the National Technical Information Service, U.S. Department of Commerce, 5285 Port Royal Road, Springfield, VA 22161. 


\section{DISCLAIMER}

Portions of this document may be illegible in electronic image products. Images are produced from the best available original document. 
KEYWORDS:

Accelerator Production of Tritium Blanket System

Conceptual Design

FLOWTRAN-TF Code

Detailed Bin Model

Safety Analysis

RETENTION - Permanent

\section{FLOWTRAN-TF CODE MODIFICATIONS MADE FOR APT BLANKET SAFETY ANALYSES}

SAVANNAH RIVER TECHNOLOGY CENTER

L. Larry Hamm

Si Young Lee

M. Andy Shadday

Frank G. Smith, III

Publication Date: July 1998

Westinghouse Savannah River Company

Savannah River Site

Aiken, SC 29808

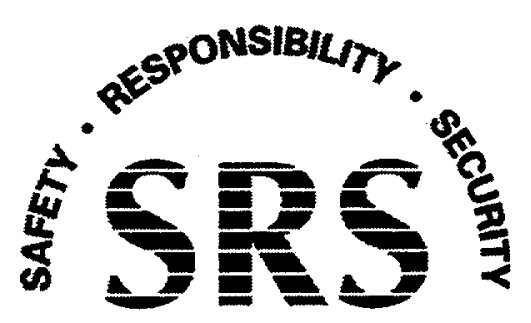

SAVANNAH RIVER SITE

Prepared for the U.S. Department of Energy under Contract No. DE-AC09-96SR18500 
DOCUMENT: WSRC-TR-98-0056

TITLE: FLOWTRAN-TF CODE MODIFICATIONS MADE FOR APT BLANKET SAFETY ANALYSES

APPROVALS

tom Tram

Date: $7-16-98$

L. Larry Hamm, Task Leader (EM\&S Group/SRTC)

Date: $7-20-98$

Si Young Lee, Co-author (EM\&S Group/SRTC)

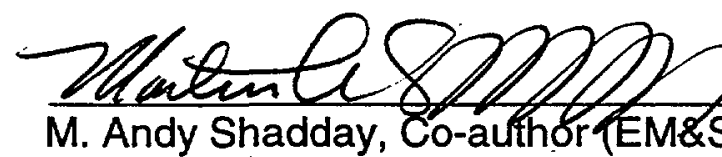

\&S Group/SRTC)

Date: $2 / 20 / 98$

Zrank 1 lmett $\$$

Frank G. Smith, III, Co-author (PC\&C Group/SRTC)

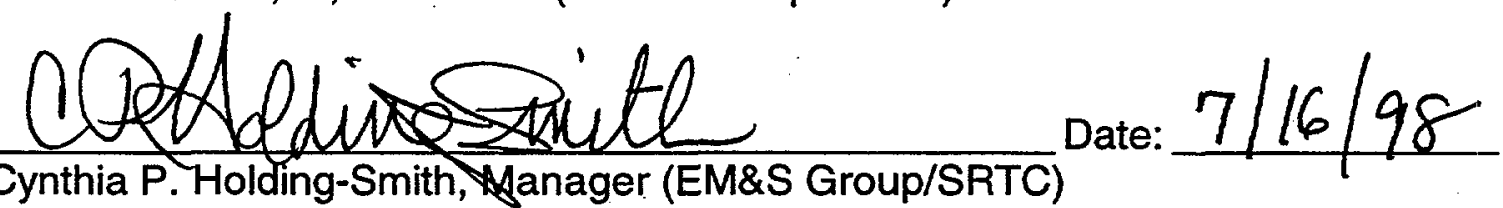

m.Q:EBra

Martha A. Ebra, Manager (EDS/SRTC)

Date: $2 / 20 / 98$

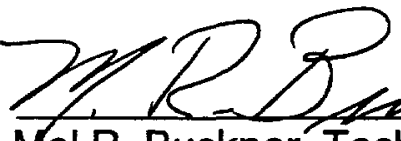

Mel R. Buckner, Technical \& Regulatory Lead (APT OPO)

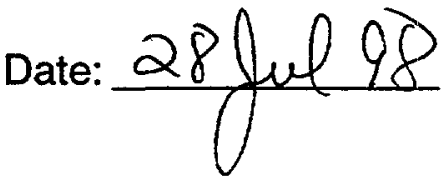

The internal technical review function is being performed at the APT project level and is coordinated through LANL. 


\section{Table of Contents}

1 Introduction 1

2 Code Modifications $\quad 1$

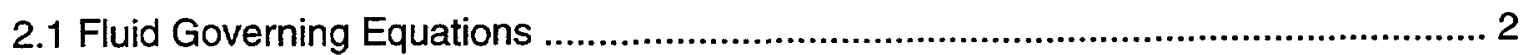

2.2 Solid Governing Equations ...................................................................... 4

3 FLOWTRAN-TF Subroutines 6

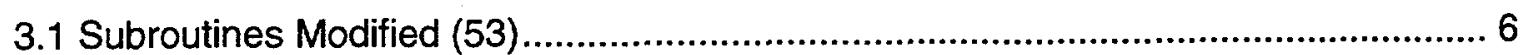

3.2 Subroutines Eliminated (20).........................................................................10

3.3 Subroutines Added for Finite Element Heat Conduction (25)..................................10

3.4 Subroutines Added for Other Purposes (11) ..........................................................11

4 Quality Assurance $\quad 12$

5 References $\quad 15$

\section{List of Figures}

Figure 2-1 Simplified schematic diagram of FLOWTRAN-TF solution logic.................. 5

Figure 4-1 Cosine axial power shape used in test case.............................................14

\section{List of Tables}

Table 2-1 Coefficients for Macbeth-Bowring Critical Heat Flux Correlation. 4

Tablé 4-1 Results of cosine power distribution test case. 
(This Page Intentionally Left Blank)

-iv- 


\section{Introduction}

This report provides background information for a series of reports documenting accident scenario simulations for the Accelerator Production of Tritium (APT) blanket heat removal systems [1-7]. The simulations were performed in support of the Preliminary Safety Analysis Report (PSAR) for the APT. This report gives a brief description of modifications made to the previously existing FLOWTRAN-TF code which was used for detailed blanket bin modeling.

Modifications made to the FLOWTRAN-TF code for safety analysis of the APT blanket region basically fall into three categories: (1) replacement of the original solid heat conduction subroutines which were restricted to cylindrical coordinates with an unstructured mesh finite element based heat conduction calculation; (2) replacement of the original lateral power distribution calculations which were also specific for cylindrical coordinates with a simple calculation of the deposited power; and (3) elimination of azimuthal fluid flow calculations from the original code. The azimuthal flow equations, which had been used to model flow across rib gaps present in the SRS reactor assemblies, contained parameters specific for flow through narrow slots. Since this configuration did not apply to the APT blanket plate module, this part of the FLOWTRAN-TF coding was eliminated for greater computational efficiency. In addition to these major changes, a number of other modifications were made to add new or enhance existing capabilities in the code. These modifications are described in this document.

\section{Code Modifications}

FLOWTRAN-TF solves conjugate heat transfer problems in a somewhat sequential fashion. As such, FLOWTRAN-TF software design separates the fluid calculations from the solid heat conduction calculations. The fluid and solid calculations are interfaced through appropriate (fluid-solid interface) models. Section 2.1 contains a discussion of the upgrades made to the fluid and interface models, while Section 2.2 contains a discussion of those upgrades made to the solid heat conduction models.

\subsection{Fluid Governing Equations}

The seven most significant modifications made to the original code (apart from the reduction to one dimensional fluid flow and the generalization of the solid from cylindrical geometry) are described below:

1. Addition of a steady-state treatment for an oxide layer on the outer surface of the metal. This was accomplished by using a steady-state approximation to adjust the surface heat transfer coefficient to an effective value that includes the effective thermal resistance of the surface oxide layer:

$$
\frac{1}{h_{\text {eff }}}=\frac{1}{h}+\frac{\Delta x_{\text {oxide }}}{k_{\text {oxide }}}
$$


where: $h_{\text {eff }}$ effective heat transfer coefficient

$h$ heat transfer coefficient without oxide layer

$\Delta x_{\text {oxide }}$ thickness of oxide layer (input to code)

$k_{\text {oxide }}$ thermal conductivity of oxide (input to code)

2. Inclusion of the Macbeth-Bowring critical heat flux correlation [8] as a code option. This correlation can be written in the form:

$$
q^{\prime \prime}=\frac{A^{\prime}+D G\left(\Delta h_{s u b}\right)_{i} / 4}{C^{\prime}+z}
$$

where $z$ is the channel length, $D$ the channel hydraulic diameter, $G$ the total mass velocity and $\left(\Delta h_{s u b}\right)_{i}$ is the inlet subcooling. The functions $A^{\prime}$ and $C^{\prime}$ are given by the expressions

$$
\begin{aligned}
& A^{\prime}=\frac{0.579 F_{1} D G h_{f g}}{1.0+0.0143 F_{2} D^{0.5} G} \\
& C^{\prime}=\frac{0.077 F_{3} D G}{1.0+0.347 F_{4}(G / 1356)^{n}} \quad n=2-0.5 P
\end{aligned}
$$

The four functions $F_{i}$ are obtained from the Bowring critical heat flux correlation for water data and can be cast into the general form:

$$
F_{i}=C_{1, i}+C_{2, i} P^{C_{3, i}} \exp \left[C_{4, i}(1-P)\right]
$$

with the additional requirement that, after the function evaluations, we set

$$
F_{2}=\frac{F_{1}}{F_{2}} \quad \text { and } \quad F_{4}=F_{3} F_{4}
$$

Constants used for evaluating the $F_{i}$ functions are listed in Table 2-1 at the end of this report. The pressure $P$ in all of the above correlations is a reduced pressure equal to the system pressure in Pascal divided by $6.895 \times 10^{6}$.

3. Utilization of the "F-factor" method to adjust the critical heat flux for a non-uniform axial heat flux profile [9]. The method corrects the estimation of critical heat flux based on a uniform heat flux distribution by dividing by the "F-factor"

$$
q_{n u}^{\prime \prime}=\frac{q_{u}^{\prime \prime}}{F}
$$

The correction factor $F$ is calculated as 


$$
F=\frac{\Omega}{1-\exp \left(-\Omega z_{\text {crit }}\right)} \int_{0}^{z_{c r i t}} \frac{q^{\prime \prime}(z)}{q^{\prime \prime}\left(z_{\text {crit }}\right)} \exp \left[-\Omega\left(z_{\text {crit }}-z\right)\right] d z
$$

where $\Omega=\frac{185.6\left(1-z_{c r i t}\right)^{4.31}}{G^{0.478}}$ with $G$ in $\mathrm{kg} /\left(\mathrm{s}-\mathrm{m}^{2}\right)$ and $z$ in meters.

The calculation is implemented by taking the axial position in the center of each fluid cell as $z_{c r i t}$ and calculating the $F$-factor for each cell by integrating the defining equation along the axial length of the channel.

4. Inclusion of criteria checking for onset of nucleate boiling, onset of significant void formation, critical heat flux, and maximum solid temperature. Onset of nucleate boiling uses the Mikic-Rohsenow boiling correlation, onset of significant void uses the Saha-Zuber correlation, and critical heat flux is selected through input to be based on either the Macbeth-Bowring correlation, Biasi correlation, or a correlation derived from data collected at SRS on aluminum surfaces. Implementations of the Mikic-Rohsenow, Saha-Zuber, Biasi, and SRS correlations in the code are unchanged from the original versions described in the FLOWTRAN-TF code manual [10].

5. Adjustment of the fluid friction factor at the wall to account for heated wall effects using:

$$
f_{w}=\frac{\hat{f}_{w}}{\sum A_{\text {wall }}} \sum\left\{A_{\text {wall }}\left(\frac{\mu_{\text {wall }}}{\mu_{b u l k}}\right)^{0.35}\right\}
$$

where $A_{\text {wall }}$ is the surface area, $\mu_{\text {wall }}$ is the fluid viscosity evaluated at the wall temperature, $\mu_{b u l k}$ is the fluid viscosity evaluated at the bulk fluid temperature and the summation is taken over all of the solid surfaces surrounding the flow channel. The equation is applied at each axial level in the flow channel.

6. Restructuring the code so that the solid heat conduction calculation is only performed after the fluid solution has converged and including the option to perform the heat conduction calculations on a different time frequency from the fluid calculations. Figure 2-1 shows a simplified schematic diagram of the basic FLOWTRAN-TF solution algorithm.

7. Addition of a subroutine to write output data files in a format compatible with the Tecplot graphics software.

In addition to the modifications listed above, numerous minor changes were made to improve some of the coding and to enhance the code structure. 


\subsection{Solid Governing Equations}

[TBA...]

Table 2-1 Coefficients for Macbeth-Bowring Critical Heat Flux Correlation.

\begin{tabular}{|c|ccrr|}
\hline Function & $C_{1}$ & $C_{2}$ & $C_{3}$ & $C_{4}$ \\
\hline$F_{1}$ & 0.478 & 0.522 & 18.942 & 20.810 \\
$F_{2}$ & 0.236 & 0.764 & 1.316 & 2.444 \\
$F_{3}$ & 0.400 & 0.600 & 17.023 & 16.658 \\
$F_{4}$ & 0.000 & 1.000 & 1.649 & 0.000 \\
\hline
\end{tabular}




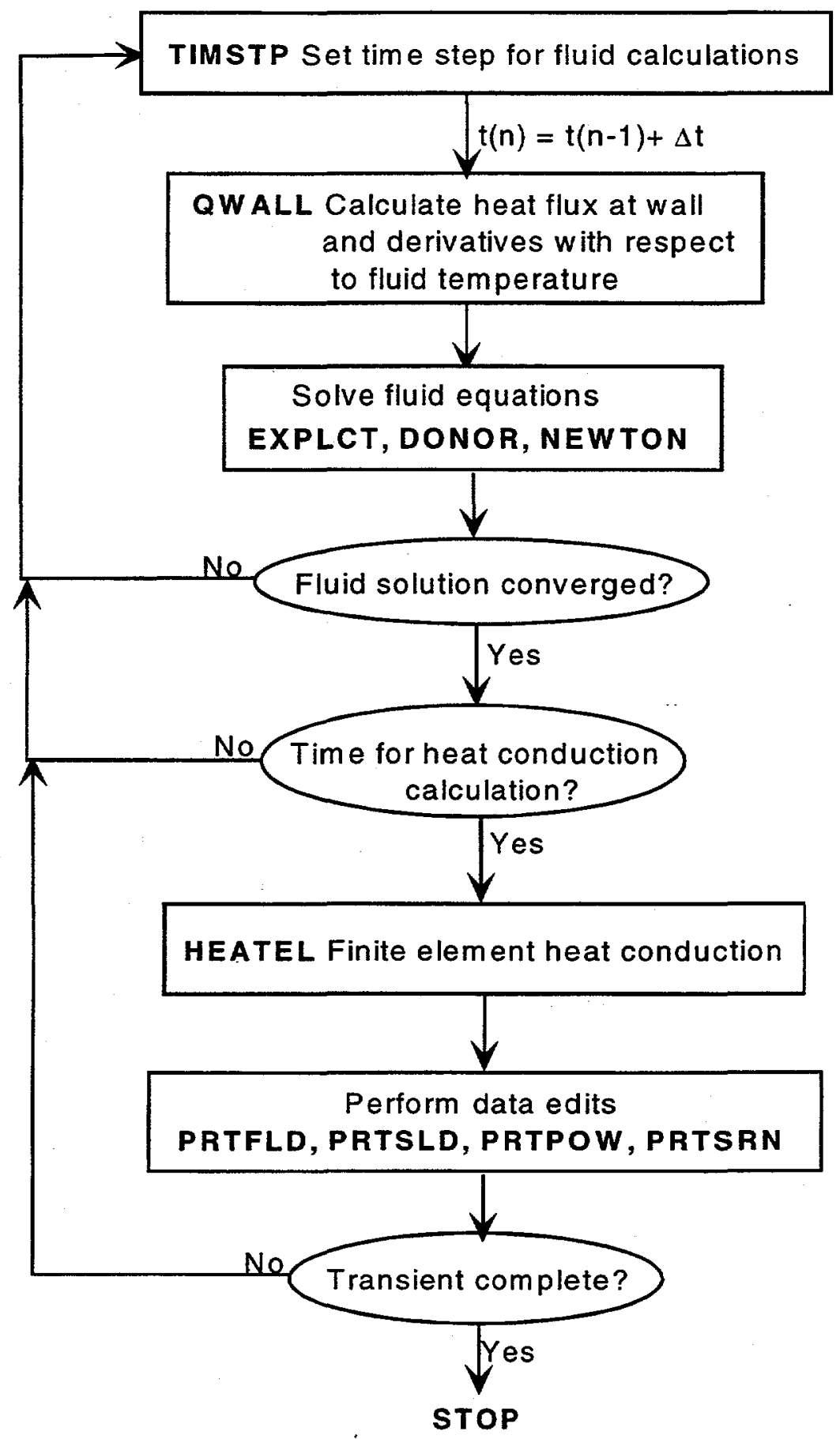

Figure 2-1 Simplified schematic diagram of FLOWTRAN-TF solution logic. 


\section{FLOWTRAN-TF Subroutines}

The revised FLOWTRAN-TF code contains 124 subroutines and 4 include files. During the course of the APT blanket model development, almost every file was modified in some way. Code modifications fall into three general areas: (1) modifications to original subroutines; (2) elimination of original subroutines; and (3) addition of new subroutines. Most of the modified subroutines reflect changes made to eliminate the azimuthal flow equations from the original version of FLOWTRAN-TF. Almost all of the subroutines eliminated from the original FLOWTRAN-TF code were specifically designed for the azimuthal rib flow, the cylindrical heat conduction solution and the power distribution calculation. Similarly, the majority of the subroutines added to the original code are used for the finite-element heat conduction calculations. The following listings name the subroutines that were changed, give a brief description of the subroutine function and, in the case of modified subroutines, briefly describe the changes made. Minor modifications that were made to simply enhance the code structure or its cosmetic appearance and did not have a significant impact on the code operations are not listed.

\subsection{Subroutines Modified (53)}

accchk - checks accuracy of fluid solution

eliminated azimuthal dependence from fluid variables

avec1 - calculates one-dimensional fluid momentum advection terms

modified to use subroutine weight

avecb - calculates fluid momentum advection in bottom section

modified to use subroutine weight

avect - calculates fluid momentum advection in top section

modified to use subroutine weight

avecz - calculates fluid momentum advection in axial flow direction

eliminated azimuthal dependence from fluid variables, eliminated azimuthal momentum calculations and modified to use subroutine weight

axialp - calculates axial power distribution

modified to use either normalized or unnormalized axial power shapes

chen - calculates surface heat flux using Chen's boiling heat transfer correlation

eliminated some derivative calculations no longer used

chf - calculates critical heat flux from user specified correlation

added Macbeth-Bowring critical heat flux correlation as an option

const - calculates fluid constitutive relations

eliminated azimuthal dependence from fluid variables

crit - evaluates criteria functions

modified to include additional criteria (ONB, OSV, CHF) 
donorb - donor differencing in bottom section of model

modified to use subroutine weight

donorm - donor differencing in middle section of model

eliminated azimuthal dependence from fluid variables and modified to use subroutine weight

donort - donor differencing in top section of model

modified to use subroutine weight

expl1d - evaluates explicit momentum terms in top and bottom sections

modified to improve calculation efficiency, no functional changes

expl2d - evaluates explicit momentum terms in middle section

eliminated azimuthal dependence from fluid variables and azimuthal momentum calculations

explct - driver routine for evaluation of explicit momentum terms

eliminated azimuthal dependence from fluid variables

explpl - evaluates explicit momentum terms in top plenum

modified to improve calculation efficiency, no functional changes

expltb - evaluates explicit momentum terms in bottom plenum

modified to improve calculation efficiency, no functional changes

geofld - sets fluid geometric parameters

eliminated azimuthal dependence from fluid variables and azimuthal geometry calculations

hconv - calculates single phase convection heat transfer correlation

modified to include oxide layer resistance

inbond - controls input of fluid boundary conditions

modified to let code estimate initial flow splits based on channel areas and to accept input of pressure, void and temperature profiles

initfd - initializes fluid calculations

eliminated azimuthal dependence from fluid variables and use input profiles

initl - controls initialization of the entire calculations

added initialization for finite element calculations removed old power initialization and made numerous modifications for subroutine calls

inner - solves the reduced fluid problem for pressure changes

eliminated azimuthal dependence from fluid variables and completely eliminated some azimuthal variables

inopts - inputs calculation options and parameters 
eliminated all azimuthal variables form the input and added additional options such as use of Macbeth-Bowring CHF correlation

input - general subroutine to control code input

added subroutines to input finite element mesh

jacobb - evaluates Jacobian for fluid pressure solution in bottom section eliminated azimuthal dependence from coefficients

jacobm - evaluates Jacobian for fluid pressure solution in middle section eliminated azimuthal dependence from coefficients and azimuthal calculations

jacobt - evaluates Jacobian for fluid pressure solution in top section eliminated azimuthal dependence from coefficients

knobs.inc - include file containing adjustable input parameters eliminated azimuthal parameters

mikic - calculates surface heat flux using Mikic-Roshenow boiling correlation eliminated rib effectiveness factor calculation

newton - solves set of non-linear fluid equations by Newton's method eliminated azimuthal dependence from fluid variables

params.inc - include file containing parameters used in calculations numerous modifications that reflect the elimination of azimuthal variables and the addition of new heat transfer and input options

point - sets pointers into a array for code vectors and matrices numerous modifications to eliminate old variables and add new ones

points.inc - include file holding array pointer values numerous modifications to eliminate old variables and add new ones propck - checks that fluid properties are within correlation ranges eliminated azimuthal dependence from fluid variables and solid property checking specific for reactro assembly metals while adding parameters for APT materials

prtfld - prints fluid solution eliminated azimuthal dependence from fluid variables and from output prtpow - prints power distribution in solid changed from cylindrical geometry to finite element mesh

prtsid - prints solid temperature solution changed from cylindrical geometry to finite element mesh 
prtsrn - prints information to screen as code is running

eliminated azimuthal dependence from fluid variables also modified to work on silicon graphics workstation

qwall - evaluates surface heat fluxes

eliminated azimuthal dependence from fluid variables, modified to calculate numerical derivatives in place of analytical values used previously, changed from cylindrical geometry to finite element mesh

reset - puts values calculated at new time step into old time arrays

eliminated azimuthal dependence from fluid variables and eliminate solid temperatures

restrt - reads data from restart file to start solution

eliminated azimuthal dependence from fluid variables and modified to use new solid geometry

setsid - sets solid parameters to begin solution

eliminated calculation of cylindrical geometry parameters and added calculation of finite element mesh volumes and areas

srihtc - calculates heat transfer coefficient based on SRS data

modified to include oxide layer resistance

state - evaluates equation of state for fluid

eliminated azimuthal dependence from fluid variables

store - stores solution for use in code restarts

eliminated azimuthal dependence from fluid variables and modified to use new solid geometry

timstp - sets calculation time step for fluid

- eliminated azimuthal dependence from fluid variables and azimuthal flow Courant limit

trans - main part of code that drives the transient calculations

numerous modifications to incorporate finite element heat conduction subroutines and revised fluid calculations

tridag - solves tri-diagonal matrix for changes in fluid pressure

original form was used in both the fluid and solid calculations

a simplified version for only the fluid solution was implemented

updatp - updates pressure solution

eliminated azimuthal dependence from fluid variables

updatv - updates velocity solution

eliminated azimuthal dependence from fluid variables 


\subsection{Subroutines Eliminated (20)}

avecx - calculates advection terms in azimuthal direction

chlski - Cholesky decomposition matrix solver

condt - evaluates metal thermal conductivity

cylndr - loops over individual cylinders for three dimensional solid heat conduction

dmpplt - writes binary plotting files

eossld - equation of state for solid materials

fwallx - evaluates wall friction in azimuthal direction

geosid - sets up cylindrical geometry for solid mesh

hfluid - calculates fluid enthalpy at saturation

indpwr - reads input for deposited power distribution

initsd - initializes solid temperatures

inpowr - reads input for deposited power distribution

pdist - calculates deposited power distribution in solid

pnorm - normalizes deposited power distribution

radshp - evaluates radial power shape in cylindrical geometry

ring - solves three dimensional solid heat conduction at each axial level

rsteam - calculates steam density at saturation

solid - driver to solve three dimensional solid heat conduction

turbx - calculates turbulent exchange across rib gaps

vrib - calculates velocity across rib gaps

\subsection{Subroutines Added for Finite Element Heat Conduction (25)}

axcon - explicit axial conduction

baksub - back substitution part of matrix solution

cauchy - applies Cauchy boundary conditions on solid mesh

decomp - performs matrix decomposition

dirhbc - applies Dirchlet boundary conditions on solid mesh

femesh - outputs finite element mesh coordinates

gauss 4 - sets quadrature points and weights for rectangular elements

gpsk - applies Gibbs-Poole-Stockmeyer matrix reordering scheme

This subroutine was adapted from algorithm 582 in the TOMS library.

gquad - performs Gaussian quadrature of surface integrals 
greduc - performs Gaussian elimination to reduce stiffness matrix

grid2d - generates two-dimensional finite element grid from input parameters

heatel - drives finite element solid heat conduction calculation

ingrid - inputs parameters used to generate two-dimensional grid

intgrl - calculates element independent volume and area integrals

Ihsmat - generates left hand side of heat conduction matrix

mematrix - evaluates left hand side local element geometry matrices

mlmatrix - evaluates left hand side local element geometry matrices

optnum - prepares input to gpsk and runs routine to apply the renumbering algorithm

rhsgen - generates right hand side of heat conduction matrix

rsurf - analytically evaluates surface integrals for Cauchy boundary conditions

rvect - evaluates local right hand side element geometry vector with power generation

setmat - sets and outputs parameters for solid heat conduction solution

shape4 - sets shape functions and derivatives for rectangular coordinates

skylin - implements skyline storage for heat conduction matrix

surfelem - analytically calculates surface integrals for Cauchy boundary conditions

\subsection{Subroutines Added for Other Purposes (11)}

chksld - checks the accuracy of the solid heat conduction solution

densty - calculates fluid density

flow - calculates fluid volumetric and mass flows

htwall - applies heated wall correction to wall friction factors

hwall - calculates wall heat transfer coefficients on outer boundaries

param.inc - include file containing some fixed parameters

props - evaluates solid physical properties

qcrit - computes ONB, OSV and CHF heat fluxes used for criteria checking

qflux - updates surface heat flux and keeps running summation

source - calculates deposited power in each solid element at each time step

tecplt - writes plot files in format compatible with Tecplot-7 software

weight - calculates weighting factors for donoring in fluid difference equations 


\section{Quality Assurance}

As part of the reactor restart effort at SRS, the original FLOWTRAN-TF code was subject to strict quality assurance requirements and configuration control. FLOWTRANTF quality assurance reference documents include:

FLOWTRAN-TF Code Development Technical and QA Plan, Rev. 0 (Flach, 1991)

FLOWTRAN-TF Software Requirements Specification, Rev. 1 (Flach, 1993)

FLOWTRAN-TF Software Test Plan, Rev. 1 (Flach, 1993)

The results from an extensive software testing program are provided in Ref. [11]. As previously stated, the principal changes made to the code were removal of azimuthal flow models from the fluid calculations and replacement of the power distribution and heat conduction calculations for cylindrical coordinates with a more general finite element calculation. To verify that these modifications were implemented correctly, several test cases were run with the revised code.

To verify that changes in the fluid calculations were correctly implemented, test input file test_31_01_m08.in documented in reference [11] was rerun with the modified code. This test case is a calculation to simulate data taken on air water downflow through a single annular channel. The liquid flow rate is $8 \mathrm{gpm}$ with a $-1 \mathrm{psig}$ inlet pressure and a 12 inch head of water on the channel outlet. The modified code gave results identical to those reported for the unmodified code providing confidence that changes made to the subroutines calculating the two-phase fluid flow did not alter code performance.

The finite element coding that was used to replace the original cylindrical heat conduction calculations was written at SRTC and has been used in various applications for several years [personal communication from L. L. Hamm]. To verify that the heat conduction was operating correctly after it was implemented into the FLOWTRAN-TF code a few simple test cases that could be readily checked against analytical calculations were run. In all cases, temperature distributions calculated with the code were close to the analytical results. For example, the cosine shaped power distribution shown in Fig. 4-1 was applied to a metal block $1.0 \mathrm{~m}$ long with a square cross sectional area $0.01 \mathrm{~m}$ by $0.01 \mathrm{~m}$. Metal properties were assigned the following values:

$$
\begin{aligned}
& \text { density ....................5000.0 kg/m }{ }^{3} \\
& \text { heat capacity ....................500.0 J/kg-K } \\
& \text { thermal conductivity ....................50.0 W/m-K. }
\end{aligned}
$$

The block was initially at a uniform temperature of $50 \mathrm{C}$ and deposited power was set to $0.934715 \mathrm{~W}$ (positive in the upper half of the block and negative in the lower half). Table 4-1 provides a comparison of the calculated temperature distribution at 3600 seconds to the analytical values. As can be seen from the table, the error in the calculated solution is very small indicating that the heat conduction calculation, including axial conduction, is correct.

As further verification that the revised FLOWTRAN-TF code is functioning correctly, a trial calculation was made of the normal operating conditions within the reference 1 
lateral Row 1 blanket plate component of the APT. Results from this calculation were then compared to those obtained at Los Alamos National Laboratory.

The two sets of calculations were matched as closely as possible by using the following input parameters:

1. total deposited power of $73.8 \mathrm{~kW}$ ( $120 \%$ normal operating power)

2. inlet water temperature of $53.2 \mathrm{C}$

3. application of the Dittus-Boelter heat transfer correlation

FLOWTRAN-TF calculations obtained a maximum aluminum temperature of $109.4 \mathrm{C}$ whereas LANL reported a value of $112 \mathrm{C}$ [12]. Considering that two completely different calculation techniques were used, this comparison was judged to be adequate to verify that the FLOWTRAN-TF code was performing correctly for the intended application.

Full QA documentation for the revised FLOWTRAN-TF code is not available at this time but will be provided as a part of the final APT PSAR blanket analysis. Additional verification and validation work for the revised code will also be performed in the future.

Table 4-1 Results of cosine power distribution test case.

\begin{tabular}{|c|ccc|}
\hline Axiallevel & Analytical Value & $\begin{array}{c}\text { FlowTRAN-TF } \\
\text { Calculated Value }\end{array}$ & \% \\
\hline 1 & 65.07 & 64.91 & -0.25 \\
2 & 63.60 & 63.45 & -0.24 \\
3 & 60.79 & 60.67 & -0.20 \\
4 & 56.93 & 56.85 & -0.14 \\
5 & 52.39 & 52.35 & -0.08 \\
6 & 47.61 & 47.63 & 0.04 \\
7 & 43.07 & 43.13 & 0.14 \\
8 & 39.21 & 39.31 & 0.26 \\
9 & 36.40 & 36.53 & 0.36 \\
10 & 34.93 & 35.07 & 0.40 \\
\hline
\end{tabular}




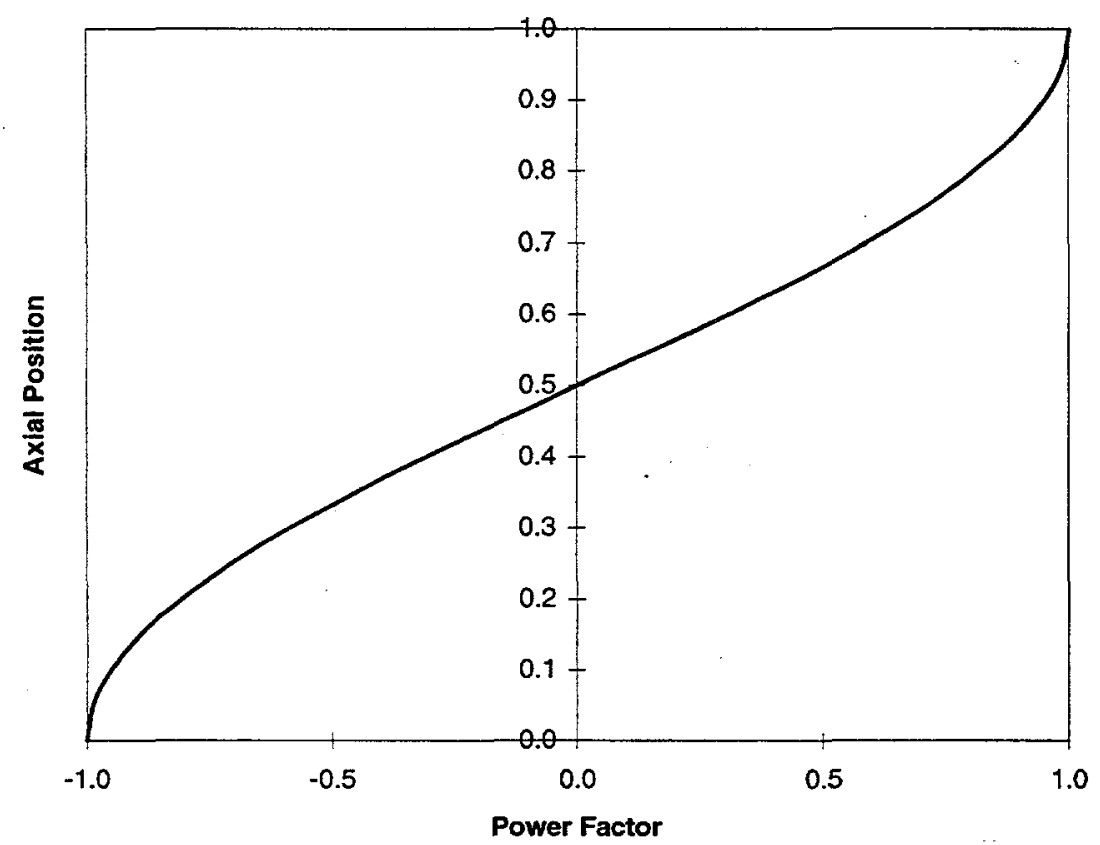

Figure 4-1 Cosine axial power shape used in test case. 


\section{References}

1. L. L. Hamm, S. Y. Lee, M. A. Shadday, and F. G. Smith, III, “APT Blanket System Loss-of-Flow Accident (LOFA) Analyses Based on Initial Conceptual Design Case 1: with Beam Shutdown and Active RHR," Westinghouse Savannah River Company, WSRC-TR-98-0058 (July 1998).

2. L. L. Hamm, S. Y. Lee, M. A. Shadday, and F. G. Smith, III, "APT Blanket System Loss-of-Coolant Accident (LOCA) Analysis Based on Initial Conceptual Design Case 1: External HR Break Near Inlet Header," Westinghouse Savannah River Company, WSRC-TR-98-0059 (July 1998).

3. L. L. Hamm, S. Y. Lee, M. A. Shadday, and F. G. Smith, III, "APT Blanket System Loss-of-Coolant Accident (LOCA) Analysis Based on Initial Conceptual Design Case 2: External HR Break at Pump Outlet with Pump Trip," Westinghouse Savannah River Company, WSRC-TR-98-0060 (July 1998).

4. L. L. Hamm, S. Y. Lee, M. A. Shadday, and F. G. Smith, III, "APT Blanket System Loss-of-Coolant Accident (LOCA) Analysis Based on Initial Conceptual Design Case 3: External HR Break at Pump Outlet without Pump Trip," Westinghouse Savannah River Company, WSRC-TR-98-0061 (July 1998).

5. L. L. Hamm, S. Y. Lee, M. A. Shadday, and F. G. Smith, III, "APT Blanket System Loss-of-Coolant Accident (LOCA) Analysis Based on Initial Conceptual Design Case 4: External Pressurizer Surge Line Break Near Inlet Header," Westinghouse Savannah River Company, WSRC-TR-98-0062 (July 1998).

6. L. L. Hamm, S. Y. Lee, M. A. Shadday, and F. G. Smith, III, "APT Blanket System Loss-of-Coolant Accident (LOCA) Analysis Based on Initial Conceptual Design Case 5: External RHR Break Near Inlet Header," Westinghouse Savannah River Company, WSRC-TR-98-0063 (July 1998).

7. L. L. Hamm, S. Y. Lee, M. A. Shadday, and F. G. Smith, III, "APT Blanket System Internally Dry Flooded Cavity Accident (IDFCA) Based on Initial Plate-Type Design - Demonstration of Bin . Heat Conduction Capability," Westinghouse Savannah River Company, WSRC-TR-98-0064 (July 1998).

8. Collier, J. G., "Convective Boiling and Condensation", 2nd Ed., McGraw-Hill, 1981, .pp. 265-267.

9. ibid, pp. 275-281.

10. Aleman, S. E., G. P. Flach, L. L. Hamm, S. Y. Lee and F. G. Smith, III, "FLOWTRAN-TF Software Design," Westinghouse Savannah River Company, WSRC-TR-92-532, (February, 1993).

11. Aleman, S. E., G. P. Flach, J. W. Jerrell, S. Y. Lee, M. A. Shaddy and F. G. Smith, III, "FLOWTRAN-TF Software Testing," WSRC-TR-93-084, Westinghouse Savannah River Company, (February, 1993).

12. Kapernick, R., "Re: Problem with First Reference Plate-Type Steady-State", e-mail message from rkapernick@lanl.gov, (December 10, 1997). 
